\title{
Research Article \\ Study of Solutions to Some Functional Differential Equations with Piecewise Constant Arguments
}

\author{
Juan J. Nieto ${ }^{1,2}$ and Rosana Rodríguez-López ${ }^{1}$ \\ ${ }^{1}$ Departamento de Análisis Matemático, Facultad de Matemáticas, Universidad de Santiago de Compostela, \\ 15782 Santiago de Compostela, Spain \\ ${ }^{2}$ Department of Mathematics, Faculty of Science, King Abdulaziz University, P.O. Box 80203, \\ Jeddah 21589, Saudi Arabia
}

Correspondence should be addressed to Rosana Rodríguez-López, rosana.rodriguez.lopez@usc.es

Received 12 October 2011; Revised 6 January 2012; Accepted 9 January 2012

Academic Editor: Josef Diblík

Copyright (C) 2012 J. J. Nieto and R. Rodríguez-López. This is an open access article distributed under the Creative Commons Attribution License, which permits unrestricted use, distribution, and reproduction in any medium, provided the original work is properly cited.

We provide optimal conditions for the existence and uniqueness of solutions to a nonlocal boundary value problem for a class of linear homogeneous second-order functional differential equations with piecewise constant arguments. The nonlocal boundary conditions include terms of the state function and the derivative of the state function. A similar nonhomogeneous problem is also discussed.

\section{Introduction}

In the study of second-order functional differential equations, there is a wide range of works dealing with periodic boundary value problems and piecewise continuous functional dependence. We mention, for instance, [1], where an equation independent of the first derivative is analyzed, and many other works as [2-14], where existence and stability results are provided.

Most of works on this field deal with nonconstructive existence results. However, in $[7,11]$, explicit solutions are found for second-order functional differential equations with piecewise constant arguments, through the calculus of the Green's function. Other works in relation with first and higher-order differential equations with delay are [15-18].

In [19], a class of linear second-order differential equations with piecewise constant arguments is considered under the nonlocal conditions $x(0)=\varphi, x(T)=x(\mu)+\psi$, where 
$\varphi, \psi \in \mathbb{R}$, that is, an initial position is assumed and the boundary conditions are independent of the derivative of the function.

In this paper, we study the following nonlocal boundary value problem for homogeneous linear second-order functional differential equations:

$$
\begin{gathered}
x^{\prime \prime}(t)+a x^{\prime}(t)+b x(t)+c x^{\prime}([t])+d x([t])=0, \quad t \in J=[0, T], \\
x(T)=x(\mu)+\varphi, \\
x^{\prime}(T)=x^{\prime}(\mu)+\psi,
\end{gathered}
$$

for $a, b, c, d, \varphi, \psi \in \mathbb{R}, T>0$ and $\mu \in(0, T)$, where the functional dependence is given by the greatest integer part $[t]$, and the nonlocal boundary conditions involve both the state function and its derivative, which is the main difference from the study in [19]. A discussion for nonhomogeneous equations is also included. We consider the existence and uniqueness of solution to this problem, providing optimal conditions and calculating the exact expression of solutions.

To better illustrate the significant differences between the results included in this paper and those in [19], we remark that [19] is devoted to the study of the same class of linear second-order differential equations with piecewise constant arguments but considering a nonlocal boundary value problem where the value of the unknown function $x$ is fixed at the initial instant $t=0$ (i.e., an initial condition is imposed) and the boundary condition also involves the value of the sought solution at the right endpoint of the interval $T$ and an intermediate point $\mu$. None of the conditions imposed in [19] involve the value of the rate of change of the solution at any points. Thus, the nonlocal conditions in [19] can be reduced, by using the expression of the solution, to a boundary value problem affecting only the state of the solution, being independent of the rate of change of the state of the system. On the other hand, the nonlocal problem considered in this paper does not fix a certain initial position and, moreover, the boundary condition introduces the dependence, not only on the state, but also on the variation of the state of the system. Indeed, the value of the unknown function and its derivative is determined by the value of the corresponding magnitudes at an intermediate point of the interval of interest. Moreover, since the results in these two papers are also extensible to the special case where the intermediate point is identical to the initial instant $(\mu=0)$, we compare the consequences of both works to explain better the implications of the study of each problem. If we consider $\mu=0$, the results in [19] are applicable to obtain the solution to a class of linear second-order differential equations with piecewise constant arguments subject to the boundary conditions $x(0)=\varphi, x(T)=x(0)+\psi=\varphi+\psi$ and, therefore, we solve a problem with fixed values of the function at the end-points of the interval. However, again for $\mu=0$, the results presented in this paper allow to characterize the existence of solution (and provide its explicit expression) imposing boundary conditions of the type $x(T)=x(0)+\varphi, x^{\prime}(T)=x^{\prime}(0)+\psi$, which include, as a particular case, periodic boundary conditions (on $x$ and $x^{\prime}$ ).

The main results are stated after a few preliminary results are recalled, and, finally, examples are included to show the applicability of these results. 


\section{Preliminaries}

Consider the equation

$$
x^{\prime \prime}(t)+a x^{\prime}(t)+b x(\mathfrak{t})+c x^{\prime}([t])+d x([t])=0, \quad t \in J=[0, T],
$$

where $a, b, c, d$ and $T>0$. The following concepts and results come from [7].

Definition 2.1. [7] Let the spaces

$$
\begin{aligned}
\Lambda:= & \{y: J \longrightarrow \mathbb{R}: y \text { is continuous in } J \backslash\{1,2, \ldots,[T]\}, \\
& \text { and there exist } \left.y\left(n^{-}\right) \in \mathbb{R}, y\left(n^{+}\right)=y(n), \forall n \in\{1,2, \ldots,[T]\}\right\}, \\
E:= & \left\{x: J \longrightarrow \mathbb{R}: x, x^{\prime} \text { are continuous and } x^{\prime \prime} \in \Lambda\right\} .
\end{aligned}
$$

A solution to (2.1) is a function $x \in E$ which satisfies (2.1), taking $x^{\prime \prime}(n)=x^{\prime \prime}\left(n^{+}\right)$, for all $n \in$ $\{0,1,2, \ldots,[T]\}$.

For the constants $a, b, c, d \in \mathbb{R}$, we define $h_{1}(s)$ as

$$
\begin{gathered}
1-\frac{d}{a} s+\frac{d}{a^{2}}\left(1-e^{-a s}\right), \quad \text { if } b=0, a \neq 0, \\
1-\frac{d}{2} s^{2}, \quad \text { if } b=0, a=0, \\
\left(1+\frac{d}{b}\right)\left(1+\frac{a}{2} s\right) e^{-(a / 2) s}-\frac{d}{b}, \quad \text { if } b \neq 0, a^{2}=4 b, \\
\left(1+\frac{d}{b}\right) \frac{\beta e^{\alpha s}-\alpha e^{\beta s}}{\beta-\alpha}-\frac{d}{b}, \quad \text { if } b \neq 0, a^{2}>4 b, \\
\left(1+\frac{d}{b}\right) e^{-(a / 2) s}\left\{\cos \sqrt{b-\frac{a^{2}}{4}} s+\frac{a}{2 \sqrt{b-a^{2} / 4}} \sin \sqrt{b-\frac{a^{2}}{4}} s\right\}-\frac{d}{b}, \quad \text { if } b \neq 0, a^{2}<4 b .
\end{gathered}
$$

Consider also $h_{2}(s)$ given by

$$
\begin{aligned}
& \frac{1}{a}\left(1-e^{-a s}-c s+\frac{c}{a}\left(1-e^{-a s}\right)\right), \quad \text { if } b=0, a \neq 0, \\
& s-\frac{c}{2} s^{2}, \quad \text { if } b=0, a=0 \\
& e^{-(a / 2) s}\left\{\frac{c}{b}\left(1+\frac{a}{2} s\right)+s\right\}-\frac{c}{b}, \quad \text { if } b \neq 0, a^{2}=4 b
\end{aligned}
$$




$$
\begin{gathered}
\frac{(\beta c / b-1) e^{\alpha s}+(1-\alpha c / b) e^{\beta s}}{\beta-\alpha}-\frac{c}{b}, \quad \text { if } b \neq 0, a^{2}>4 b, \\
e^{-(a / 2) s}\left\{\frac{c}{b} \cos \sqrt{b-\frac{a^{2}}{4}} s+\frac{1+a c /(2 b)}{\sqrt{b-a^{2} / 4}} \sin \sqrt{b-\frac{a^{2}}{4}} s\right\}-\frac{c}{b}, \quad \text { if } b \neq 0, a^{2}<4 b .
\end{gathered}
$$

In the definitions of functions $h_{1}$ and $h_{2}$, we denote, for $b \neq 0, a^{2}>4 b$,

$$
\alpha=-\frac{a}{2}+\sqrt{\left(\frac{a}{2}\right)^{2}-b}, \quad \beta=-\frac{a}{2}-\sqrt{\left(\frac{a}{2}\right)^{2}-b} .
$$

It is easy to check [7] that $h_{1}(0)=1, h_{2}(0)=0, h_{1}^{\prime}(0)=0, h_{2}^{\prime}(0)=1$.

Theorem 2.2 (see [7, Theorem 2.1]). The initial value problem

$$
\begin{gathered}
v^{\prime \prime}(t)+a v^{\prime}(t)+b v(t)+c v^{\prime}([t])+d v([t])=0, \quad t \in[0,+\infty), \\
v(0)=v_{0}, \\
v^{\prime}(0)=v_{0}^{\prime}
\end{gathered}
$$

for $v_{0}, v_{0}^{\prime} \in \mathbb{R}$, has the solution

$$
v(t)=\left(\begin{array}{ll}
h_{1}(t-n) & h_{2}(t-n)
\end{array}\right)\left(\begin{array}{ll}
C_{1} & C_{2} \\
C_{1}^{\prime} & C_{2}^{\prime}
\end{array}\right)^{n}\left(\begin{array}{l}
v_{0} \\
v_{0}^{\prime}
\end{array}\right), \quad t \in[n, n+1),
$$

where $n \in \mathbb{Z}^{+}$,

$$
\begin{array}{ll}
C_{1}=h_{1}(1), & C_{2}=h_{2}(1), \\
C_{1}^{\prime}=h_{1}^{\prime}(1), & C_{2}^{\prime}=h_{2}^{\prime}(1) .
\end{array}
$$

To simplify calculus, for $z \in[0,1]$, one denotes

$$
\begin{aligned}
H(z) & =\left(\begin{array}{ll}
h_{1}(z) & h_{2}(z) \\
h_{1}^{\prime}(z) & h_{2}^{\prime}(z)
\end{array}\right), \\
C=H(1) & =\left(\begin{array}{ll}
C_{1} & C_{2} \\
C_{1}^{\prime} & C_{2}^{\prime}
\end{array}\right) .
\end{aligned}
$$




\section{Main Results}

First, we consider the nonlocal boundary value problem

$$
\begin{aligned}
x^{\prime \prime}(t)+a x^{\prime}(t)+b x(t)+c x^{\prime}([t])+d x([t])=0, \quad t \in J=[0, T], \\
x(T)=x(\mu)+\varphi, \\
x^{\prime}(T)=x^{\prime}(\mu)+\psi,
\end{aligned}
$$

for $a, b, c, d, \varphi, \psi \in \mathbb{R}, T>0$ and $\mu \in(0, T)$.

If $\mu=0=\varphi$, the condition $x(T)=x(\mu)+\varphi$ is reduced to a periodic boundary condition of Dirichlet type. On the other hand, if $\mu=0$, we obtain $x^{\prime}(T)=x^{\prime}(0)+\psi$, and, moreover, if $\psi=0$, we deduce the periodicity of the derivative of the function.

Theorem 3.1. If $T>0$ and $\mu \in(0, T)$, then problem (3.1) is solvable for each $\left(\begin{array}{c}\varphi \\ \psi\end{array}\right)$ in the image of the mapping

$$
\mathcal{F}:\left(\begin{array}{l}
u \\
v
\end{array}\right) \longrightarrow\left[H(T-[T]) C^{[T]-[\mu]}-H(\mu-[\mu])\right] C^{[\mu]}\left(\begin{array}{l}
u \\
v
\end{array}\right)
$$

Hence, there exists a unique solution to (3.1) for every $\left(\begin{array}{c}\varphi \\ \psi\end{array}\right) \in \mathbb{R}^{2}$ if and only if the matrix

$$
\left[H(T-[T]) C^{[T]-[\mu]}-H(\mu-[\mu])\right] C^{[\mu]}
$$

is nonsingular. In this case, the solution is given by

$$
v(t)=\left(\begin{array}{ll}
h_{1}(t-n) & h_{2}(t-n)
\end{array}\right) C^{n}\left(\begin{array}{l}
v_{0} \\
v_{0}^{\prime}
\end{array}\right), \quad t \in[n, n+1), n \in \mathbb{Z}^{+},
$$

where $\left(\begin{array}{c}v_{0} \\ v_{0}^{\prime}\end{array}\right)$ is the inverse image of $\left(\begin{array}{c}\varphi \\ \psi\end{array}\right)$ by $\mathcal{F}$.

If the matrix (3.3) is singular, then there exists an infinite number of solutions to problem (3.1) for every $\left(\begin{array}{c}\varphi \\ \psi\end{array}\right)$ in the image of $\boldsymbol{\mathcal { F }}$, taking as initial position and initial slope in (3.4) any preimage of $\left(\begin{array}{c}\varphi \\ \psi\end{array}\right)$ by $\mathcal{F}$, and there exist no solutions for the remaining pairs $\left(\begin{array}{c}\varphi \\ \psi\end{array}\right) \in \mathbb{R}^{2}$.

Proof. We consider the initial value problem

$$
\begin{gathered}
v^{\prime \prime}(t)+a v^{\prime}(t)+b v(t)+c v^{\prime}([t])+d v([t])=0, \quad t \in[0,+\infty) \\
v(0)=v_{0} \\
v^{\prime}(0)=v_{0}^{\prime}
\end{gathered}
$$


for $v_{0}, v_{0}^{\prime} \in \mathbb{R}$, whose solution is, by Theorem 2.2 [7, Theorem 2.1],

$$
v(t)=\left(\begin{array}{ll}
h_{1}(t-n) & h_{2}(t-n)
\end{array}\right) C^{n}\left(\begin{array}{c}
v_{0} \\
v_{0}^{\prime}
\end{array}\right), \quad t \in[n, n+1),
$$

where $n \in \mathbb{Z}^{+}$. We analyze under which conditions the boundary conditions are fulfilled, taking into account that

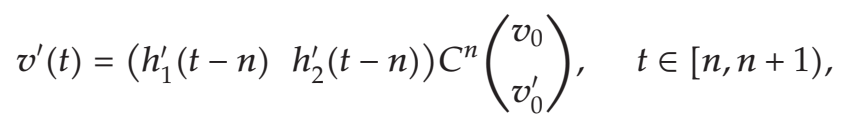

where $n \in \mathbb{Z}^{+}$.

First, we consider the case $T \notin \mathbb{Z}$, and $\mu \notin \mathbb{Z}$. To obtain the solution to (3.1), we calculate $v_{0}$ and $v_{0}^{\prime}$ from the expressions of (3.6) and (3.7), in order to satisfy $v(T)=v(\mu)+\varphi$ and $v^{\prime}(T)=v^{\prime}(\mu)+\psi$. Hence the boundary conditions are written as

$$
\begin{aligned}
& {\left[\begin{array}{lll}
\left(h_{1}(T-[T])\right. & \left.h_{2}(T-[T])\right) C^{[T]-[\mu]}-\left(h_{1}(\mu-[\mu])\right. & \left.h_{2}(\mu-[\mu])\right)
\end{array}\right] C^{[\mu]}\left(\begin{array}{l}
v_{0} \\
v_{0}^{\prime}
\end{array}\right)=\varphi,} \\
& {\left[\left(h_{1}^{\prime}(T-[T]) h_{2}^{\prime}(T-[T])\right) C^{[T]-[\mu]}-\left(h_{1}^{\prime}(\mu-[\mu]) h_{2}^{\prime}(\mu-[\mu])\right)\right] C^{[\mu]}\left(\begin{array}{c}
v_{0} \\
v_{0}^{\prime}
\end{array}\right)=\psi,}
\end{aligned}
$$

that is,

$$
\left[H(T-[T]) C^{[T]-[\mu]}-H(\mu-[\mu])\right] C^{[\mu]}\left(\begin{array}{c}
v_{0} \\
v_{0}^{\prime}
\end{array}\right)=\left(\begin{array}{c}
\varphi \\
\psi
\end{array}\right) .
$$

The properties of functions $h_{1}$ and $h_{2}$ produce that the boundary conditions, in the cases where $T \in \mathbb{Z}$, or $\mu \in \mathbb{Z}$ (or both), can be derived from the expression obtained in the case studied $T \notin \mathbb{Z}$, and $\mu \notin \mathbb{Z}$.

Therefore, this system has a solution only for $\left(\begin{array}{l}\varphi \\ \psi\end{array}\right)$ in the image of the mapping

$$
\left(\begin{array}{l}
u \\
v
\end{array}\right) \longrightarrow\left[H(T-[T]) C^{[T]-[\mu]}-H(\mu-[\mu])\right] C^{[\mu]}\left(\begin{array}{l}
u \\
v
\end{array}\right)
$$

Therefore, the solution is unique if the matrix (3.3) is nonsingular, in which case, the initial condition and initial slope are given as

$$
\begin{aligned}
\left(\begin{array}{c}
v_{0} \\
v_{0}^{\prime}
\end{array}\right) & =\left(\left[H(T-[T]) C^{[T]-[\mu]}-H(\mu-[\mu])\right] C^{[\mu]}\right)^{-1}\left(\begin{array}{c}
\varphi \\
\psi
\end{array}\right) \\
& =\left(C^{[\mu]}\right)^{-1}\left[H(T-[T]) C^{[T]-[\mu]}-H(\mu-[\mu])\right]^{-1}\left(\begin{array}{c}
\varphi \\
\psi
\end{array}\right) .
\end{aligned}
$$


On the other hand, if the matrix (3.3) is singular, for $\left(\begin{array}{c}\varphi \\ \psi\end{array}\right)$ in the image of $\mathcal{F}$, the infinitely many solutions are calculated from (3.6) taking as initial conditions any preimage of $\left(\begin{array}{c}\varphi \\ \psi\end{array}\right)$ by $\mathcal{F}$, which proves the result.

Remark 3.2. The existence and uniqueness condition (3.3) is reduced, if $[\mu]>0$, to the nonsingularity of the matrices $C$ and

$$
\left[H(T-[T]) C^{[T]-[\mu]}-H(\mu-[\mu])\right] .
$$

On the other hand, if $[\mu]=0$, it is just reduced to the nonsingularity of the matrix $[H(T-$ $\left.[T]) C^{[T]}-H(\mu)\right]$.

Remark 3.3. In Theorem 3.1, if we consider $\mu=0$, then the boundary conditions are $x(T)=$ $x(0)+\varphi, x^{\prime}(T)=x^{\prime}(0)+\psi$, and the condition of existence and uniqueness of solution is reduced to the nonsingularity of

$$
\left[H(T-[T]) C^{[T]}-I\right]
$$

which coincides with condition (15) in [7, Theorem 2.2]. Moreover, if $\varphi=\psi=0$, the abovementioned nonsingularity condition provides that the unique solution to the homogeneous equation subject to periodic boundary value conditions is the trivial solution (see [7, Theorem 2.2]).

Remark 3.4. In Theorem 3.1, the order 2 matrix (3.3) can be written in a simplified manner in the following particular cases.

(i) If $T \notin \mathbb{Z}$ and $T-[T]=\mu-[\mu]$,

$$
H(T-[T])\left[C^{[T]-[\mu]}-I\right] C^{[\mu]} .
$$

(ii) If $T \notin \mathbb{Z}$ and $\mu \in \mathbb{Z}$,

$$
\left[H(T-[T]) C^{[T]-\mu}-I\right] C^{\mu} .
$$

(iii) If $T \in \mathbb{Z}$ and $\mu \notin \mathbb{Z}$,

$$
\left[C^{T-[\mu]}-H(\mu-[\mu])\right] C^{[\mu]} .
$$

(iv) If $T, \mu \in \mathbb{Z}$,

$$
\left[C^{T-\mu}-I\right] C^{\mu} .
$$


Remark 3.5. Summarizing, the study of the solvability of (3.1) is reduced to the discussion of system (3.9). In the case of existence of an infinite number of solutions, we must analyze the rank of the matrix in (3.3) to determine whether any pair is admissible as initial position and slope or the space of initial conditions is one-dimensional. If the rank of (3.3) is zero, problem (3.1) is solvable only for $\left(\begin{array}{l}\varphi \\ \psi\end{array}\right)=\left(\begin{array}{l}0 \\ 0\end{array}\right)$, that is, problem

$$
\begin{aligned}
x^{\prime \prime}(t)+a x^{\prime}(t)+b x(t)+c x^{\prime}([t])+d x([t])=0, \quad t \in J=[0, T], \\
x(T)=x(\mu), \\
x^{\prime}(T)=x^{\prime}(\mu),
\end{aligned}
$$

for $a, b, c, d \in \mathbb{R}, T>0$ and $\mu \in(0, T)$, has an infinite number of solutions, given by (3.4), for any initial point $\left(\begin{array}{c}v_{0} \\ v_{0}^{\prime}\end{array}\right) \in \mathbb{R}^{2}$.

On the other hand, and denoting by $G$ the matrix in (3.3), if $\operatorname{rank}(G)=1$ and $\left(\begin{array}{l}\varphi \\ \psi\end{array}\right)$ depends linearly on each column of $G$, then we have a one-dimensional space of solutions, whose starting conditions $V_{0}=\left(\begin{array}{c}v_{0} \\ v_{0}^{\prime}\end{array}\right)$ are determined from the row of the system $G\left(\begin{array}{c}v_{0} \\ v_{0}^{\prime}\end{array}\right)=\left(\begin{array}{l}\varphi \\ \psi\end{array}\right)$ corresponding to a nonzero minor of $G$.

Next, with the purpose of extending Theorem 3.1 to the nonhomogeneous case, we consider the following nonlocal boundary value problem for a nonhomogeneous equation:

$$
\begin{gathered}
x^{\prime \prime}(t)+a x^{\prime}(t)+b x(t)+c x^{\prime}([t])+d x([t])=\sigma(t), \quad t \in J=[0, T], \\
x(T)=x(\mu)+\varphi, \\
x^{\prime}(T)=x^{\prime}(\mu)+\psi,
\end{gathered}
$$

for $a, b, c, d, \varphi, \psi \in \mathbb{R}, T>0, \mu \in(0, T)$, and $\sigma \in \Lambda$. Using the expression of the solution for the corresponding initial value problem provided by [19, Theorem 5.2], we prove the following existence (and uniqueness) result.

Theorem 3.6. Consider $a, b, c, d \in \mathbb{R}, T>0, \mu \in(0, T), \sigma \in \Lambda$ and $\varphi, \psi \in \mathbb{R}$. Then problem (3.19) has a unique solution if and only if the matrix (3.3) is nonsingular. Under this assumption, the unique solution to (3.19) is given by

$$
\begin{aligned}
x(t)= & \left(h_{1}(t-n) h_{2}(t-n)\right) C^{n} V_{0} \\
& +\sum_{k=0}^{n-1} \int_{k}^{k+1}\left(h_{1}(t-n) h_{2}(t-n)\right) C^{n-1-k}\left(\begin{array}{c}
g(k+1-s) \\
g^{\prime}(k+1-s)
\end{array}\right) \sigma(s) d s \\
& +\int_{n}^{t} g(t-s) \sigma(s) d s, \quad t \in[n, n+1), n \in \mathbb{Z}^{+},
\end{aligned}
$$


with $g$ defined as

$$
g(z)= \begin{cases}\frac{1}{a}\left(1-e^{-a z}\right), & \text { if } b=0, a \neq 0 \\ z, & \text { if } b=0, a=0 \\ z e^{-(a / 2) z,} & \text { if } b \neq 0, a^{2}=4 b \\ \frac{e^{\beta z}-e^{\alpha z}}{\beta-\alpha}, & \text { if } b \neq 0, a^{2}>4 b \\ \frac{1}{\sqrt{b-a^{2} / 4}} e^{-(a / 2) z} \sin \sqrt{b-\frac{a^{2}}{4}} z, & \text { if } b \neq 0, a^{2}<4 b\end{cases}
$$

and taking, as the initial condition $V_{0}$,

$$
V_{0}=\left[H(T-[T]) C^{[T]}-H(\mu-[\mu]) C^{[\mu]}\right]^{-1}\left(\left(\begin{array}{l}
\varphi \\
\psi
\end{array}\right)-\mathcal{M}_{T, \mu, \sigma}\right),
$$

where

$$
\begin{aligned}
\mathcal{M}_{T, \mu, \sigma}= & \sum_{k=0}^{[\mu]-1} \int_{k}^{k+1}\left[H(T-[T]) C^{[T]-[\mu]}-H(\mu-[\mu])\right] C^{[\mu]-1-k}\left(\begin{array}{c}
g(k+1-s) \\
g^{\prime}(k+1-s)
\end{array}\right) \sigma(s) d s \\
& +\sum_{k=[\mu]}^{[T]-1} \int_{k}^{k+1} H(T-[T]) C^{[T]-1-k}\left(\begin{array}{c}
g(k+1-s) \\
g^{\prime}(k+1-s)
\end{array}\right) \sigma(s) d s \\
& +\int_{[T]}^{T}\left(\begin{array}{c}
g(T-s) \\
g^{\prime}(T-s)
\end{array}\right) \sigma(s) d s-\int_{[\mu]}^{\mu}\left(\begin{array}{c}
g(\mu-s) \\
g^{\prime}(\mu-s)
\end{array}\right) \sigma(s) d s .
\end{aligned}
$$

On the other hand, if the matrix (3.3) is singular, the number of solutions to (3.19) is determined by the discussion of the linear system

$$
\left[H(T-[T]) C^{[T]}-H(\mu-[\mu]) C^{[\mu]}\right] V_{0}=\left(\left(\begin{array}{l}
\varphi \\
\psi
\end{array}\right)-\mathcal{M}_{T, \mu, \sigma}\right)
$$

and, for each solution $V_{0}$ to this system (in case it exists), the expression (3.20) provides a solution to (3.19). 
Proof. The result follows from the expression of the solution (3.20) for the corresponding initial value problem, whose derivative is given by

$$
\begin{aligned}
x^{\prime}(t)= & \left(h_{1}^{\prime}(t-n) \quad h_{2}^{\prime}(t-n)\right) C^{n} V_{0} \\
& +\sum_{k=0}^{n-1} \int_{k}^{k+1}\left(h_{1}^{\prime}(t-n) \quad h_{2}^{\prime}(t-n)\right) C^{n-1-k}\left(\begin{array}{c}
g(k+1-s) \\
g^{\prime}(k+1-s)
\end{array}\right) \sigma(s) d s \\
& +\int_{n}^{t} g^{\prime}(t-s) \sigma(s) d s, \quad t \in[n, n+1), n \in \mathbb{Z}^{+},
\end{aligned}
$$

and the fact that the restrictions represented by the boundary conditions produce, respectively,

$$
\begin{aligned}
& \left(\begin{array}{ll}
1 & 0
\end{array}\right)\left[H(T-[T]) C^{[T]}-H(\mu-[\mu]) C^{[\mu]}\right] V_{0}=\varphi-\left(\begin{array}{ll}
1 & 0
\end{array}\right) \mathcal{M}_{T, \mu, \sigma} \\
& \left(\begin{array}{ll}
0 & 1
\end{array}\right)\left[H(T-[T]) C^{[T]}-H(\mu-[\mu]) C^{[\mu]}\right] V_{0}=\psi-\left(\begin{array}{ll}
0 & 1
\end{array}\right) \mathcal{M}_{T, \mu, \sigma}
\end{aligned}
$$

\section{Examples}

We present some examples where different situations are analyzed in order to decide if the existence and uniqueness condition (nonsingularity of (3.3)) holds and, in case of nonuniqueness, the dimension of the space of solutions. The exact expression of the solution (or solutions) is given explicitly.

We recall that, for $s \in[0,1], H(s)$ is given, depending on the values of the coefficients $a, b, c, d$ as follows.

(i) If $b=0, a \neq 0$,

$$
H(s)=\left(\begin{array}{cc}
1-\frac{d}{a} s+\frac{d}{a^{2}}\left(1-e^{-a s}\right) & \frac{1}{a}\left(1-e^{-a s}-c s+\frac{c}{a}\left(1-e^{-a s}\right)\right) \\
\frac{d}{a}\left(-1+e^{-a s}\right) & \frac{1}{a}\left((a+c) e^{-a s}-c\right)
\end{array}\right)
$$

thus

$$
C=H(1)=\left(\begin{array}{ll}
C_{1} & C_{2} \\
C_{1}^{\prime} & C_{2}^{\prime}
\end{array}\right)=\left(\begin{array}{cc}
1-\frac{d}{a}+\frac{d}{a^{2}}\left(1-e^{-a}\right) & \frac{1}{a}\left(1-e^{-a}-c+\frac{c}{a}\left(1-e^{-a}\right)\right) \\
\frac{d}{a}\left(-1+e^{-a}\right) & \frac{1}{a}\left((a+c) e^{-a}-c\right)
\end{array}\right)
$$


Note that the determinant of the matrix $C$ is equal to

$$
\operatorname{det}(C)=\frac{e^{-a}\left(\left(e^{a}-a-1\right) d+\left(a-a e^{a}\right) c+a^{2}\right)}{a^{2}} .
$$

(ii) If $b=0, a=0$,

$$
H(s)=\left(\begin{array}{cc}
1-\frac{d}{2} s^{2} & s-\frac{c}{2} s^{2} \\
-d s & 1-c s
\end{array}\right),
$$

thus

$$
C=H(1)=\left(\begin{array}{ll}
C_{1} & C_{2} \\
C_{1}^{\prime} & C_{2}^{\prime}
\end{array}\right)=\left(\begin{array}{cc}
1-\frac{d}{2} & 1-\frac{c}{2} \\
-d & 1-c
\end{array}\right) .
$$

The determinant of the matrix $C$ is equal to

$$
\operatorname{det}(C)=\frac{d}{2}-c+1
$$

(iii) If $b \neq 0, a^{2}=4 b$,

$$
H(s)=\left(\begin{array}{cc}
\left(1+\frac{d}{b}\right)\left(1+\frac{a}{2} s\right) e^{-(a / 2) s}-\frac{d}{b} e^{-(a / 2) s}\left\{\frac{c}{b}\left(1+\frac{a}{2} s\right)+s\right\}-\frac{c}{b} \\
\left(1+\frac{d}{b}\right)\left(-\frac{a^{2}}{4} s\right) e^{-(a / 2) s} & e^{-(a / 2) s}\left\{\frac{-a^{2} c}{4 b} s-\frac{a}{2} s+1\right\}
\end{array}\right),
$$

thus

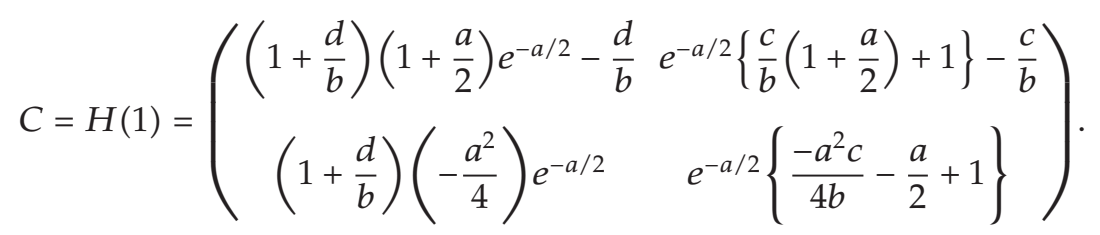

The determinant of the matrix $C$ is equal to

$$
\operatorname{det}(C)=e^{-a} \frac{\left((2 a-4) e^{a / 2}+4\right) d-a^{2} e^{a / 2} c+4 b}{4 b}
$$


(iv) If $b \neq 0, a^{2}>4 b$, and denoting

$$
H(s)=\left(\begin{array}{cc}
\alpha=-\frac{a}{2}+\sqrt{\left(\frac{a}{2}\right)^{2}-b}, & \beta=-\frac{a}{2}-\sqrt{\left(\frac{a}{2}\right)^{2}-b} \\
\left(1+\frac{d}{b}\right) \frac{\beta e^{\alpha s}-\alpha e^{\beta s}}{\beta-\alpha}-\frac{d}{b} & \frac{(\beta c / b-1) e^{\alpha s}+(1-\alpha c / b) e^{\beta s}}{\beta-\alpha}-\frac{c}{b} \\
(b+d) \frac{e^{\alpha s}-e^{\beta s}}{\beta-\alpha} & \frac{(c-\alpha) e^{\alpha s}+(\beta-c) e^{\beta s}}{\beta-\alpha}
\end{array}\right),
$$

thus

$$
C=H(1)=\left(\begin{array}{cc}
\left(1+\frac{d}{b}\right) \frac{\beta e^{\alpha}-\alpha e^{\beta}}{\beta-\alpha}-\frac{d}{b} & \frac{(\beta c / b-1) e^{\alpha}+(1-\alpha c / b) e^{\beta}}{\beta-\alpha}-\frac{c}{b} \\
\left(1+\frac{d}{b}\right) \frac{b\left(e^{\alpha}-e^{\beta}\right)}{\beta-\alpha} & \frac{(c-\alpha) e^{\alpha}+(\beta-c) e^{\beta}}{\beta-\alpha}
\end{array}\right)
$$

In this case, the determinant of the matrix $C$ is equal to

$$
\begin{aligned}
\operatorname{det}(C)= & \left(1+\frac{d}{b}\right)\left(\frac{\left(\beta e^{\alpha}-\alpha e^{\beta}\right)\left((c-\alpha) e^{\alpha}+(\beta-c) e^{\beta}\right)}{(\beta-\alpha)^{2}}-\frac{\left(e^{\alpha}-e^{\beta}\right)(\beta c-b) e^{\alpha}+(b-\alpha c) e^{\beta}}{(\beta-\alpha)^{2}}\right) \\
& -\frac{d}{b} \frac{(c-\alpha) e^{\alpha}+(\beta-c) e^{\beta}}{\beta-\alpha}+c\left(1+\frac{d}{b}\right) \frac{e^{\alpha}-e^{\beta}}{\beta-\alpha} \\
= & \left(1+\frac{d}{b}\right) \frac{\alpha^{2}+\beta^{2}-2 b}{(\beta-\alpha)^{2}} e^{\alpha+\beta}+\frac{1}{\beta-\alpha}\left(\frac{d}{b} \alpha e^{\alpha}-\frac{d}{b} \beta e^{\beta}+c e^{\alpha}-c e^{\beta}\right) \\
= & \left(1+\frac{d}{b}\right) e^{-a}+\frac{1}{\beta-\alpha}\left(\left(\frac{d}{b} \alpha+c\right) e^{\alpha}-\left(\frac{d}{b} \beta+c\right) e^{\beta}\right) .
\end{aligned}
$$

(v) If $b \neq 0, a^{2}<4 b$, and denoting $R=\sqrt{b-a^{2} / 4}$,

$$
H(s)=\left(\begin{array}{cc}
\left(1+\frac{d}{b}\right) e^{-(a / 2) s}\left\{\cos R s+\frac{a}{2 R} \sin R s\right\}-\frac{d}{b} e^{-(a / 2) s}\left\{\frac{c}{b} \cos R s+\frac{1+a c /(2 b)}{R} \sin R s\right\}-\frac{c}{b} \\
-\frac{1}{R}(b+d) e^{-(a / 2) s} \sin R s & e^{-(a / 2) s}\left\{\cos R s-\frac{a+2 c}{2 R} \sin R s\right\}
\end{array}\right)
$$


thus

$$
C=H(1)=\left(\begin{array}{cc}
\left(1+\frac{d}{b}\right) e^{-a / 2}\left\{\cos R+\frac{a}{2 R} \sin R\right\}-\frac{d}{b} e^{-a / 2}\left\{\frac{c}{b} \cos R+\frac{1+a c /(2 b)}{R} \sin R\right\}-\frac{c}{b} \\
-\frac{1}{R}(b+d) e^{-a / 2} \sin R & e^{-a / 2}\left\{\cos R-\frac{a+2 c}{2 R} \sin R\right\}
\end{array}\right) .
$$

The determinant of $C$ is given by

$$
\begin{aligned}
\operatorname{det}(C)= & \left(1+\frac{d}{b}\right) e^{-a}\left\{\cos ^{2} R-\frac{c}{R} \sin R \cos R-\frac{a(a+2 c)}{4 R^{2}} \sin ^{2} R\right\} \\
& -\frac{d}{b} e^{-a / 2} \cos R+\frac{d}{b} \frac{a+2 c}{2 R} \sin R+\frac{1}{R}\left(1+\frac{d}{b}\right) c e^{-a} \sin R \cos R \\
& +\frac{b+d}{R^{2}} e^{-a}\left(1+\frac{a c}{2 b}\right) \sin ^{2} R-\frac{c}{R}\left(1+\frac{d}{b}\right) e^{-a / 2} \sin R \\
= & \left(1+\frac{d}{b}\right) e^{-a} \cos ^{2} R+\left(1+\frac{d}{b}\right) e^{-a}\left(-\frac{a(a+2 c)}{4 R^{2}}+\frac{b}{R^{2}}\left(1+\frac{a c}{2 b}\right)\right) \sin ^{2} R \\
& -\frac{d}{b} e^{-a / 2} \cos R+\frac{1}{R}\left(\frac{d(a+2 c)}{2 b}-c\left(1+\frac{d}{b}\right) e^{-a / 2}\right) \sin R \\
= & \left(1+\frac{d}{b}\right) e^{-a}-\frac{d}{b} e^{-a / 2} \cos R+\frac{1}{R}\left(\frac{d(a+2 c)}{2 b}-c\left(1+\frac{d}{b}\right) e^{-a / 2}\right) \sin R
\end{aligned}
$$

Example 4.1. Consider the problem

$$
\begin{aligned}
x^{\prime \prime}(t)+x^{\prime}(t)+d x([t])=0, \quad t \in J=\left[0, \frac{3}{2}\right], \\
x\left(\frac{3}{2}\right)=x\left(\frac{1}{2}\right)+\varphi, \\
x^{\prime}\left(\frac{3}{2}\right)=x^{\prime}\left(\frac{1}{2}\right)+\psi,
\end{aligned}
$$

where $d, \varphi, \psi \in \mathbb{R}$. In this case, $b=c=0, a=1 \neq 0, T=3 / 2$ and $\mu=1 / 2$. Therefore, we get $H(s)=\left(\begin{array}{cc}d\left(1-e^{-s}\right)-d s+1 & 1-e^{-s} \\ d e^{-s}-d & e^{-s}\end{array}\right)$ so that matrix $G=\left[H(T-[T]) C^{[T]-[\mu]}-H(\mu-[\mu])\right] C^{[\mu]}=$ $H(1 / 2)[C-I]$ is reduced to

$$
G=\left(\begin{array}{cc}
-\frac{e^{-3 / 2}\left(\sqrt{e}\left(d^{2}+2 e d\right)-2 d^{2}+(2-2 e) d\right)}{2} & \frac{e^{-3 / 2}(\sqrt{e}(e-1) d+(2-2 e) d+2 e-2)}{2} \\
e^{-3 / 2}\left(\sqrt{e} d^{2}-d^{2}+(1-e) d\right) & -e^{-3 / 2}(\sqrt{e}(e-1) d+(1-e) d+e-1)
\end{array}\right)
$$


whose determinant is calculated as

$$
\begin{aligned}
\operatorname{det}(G) & =-\frac{e^{-2}\left(\sqrt{e}\left((3 e-3) d^{2}+(2-2 e) d\right)+\left(2 e-2 e^{2}\right) d^{2}\right)}{2} \\
& =-\frac{\left(e^{-3 / 2}(3 e-3)+2 e^{-1}-2\right) d^{2}+e^{-3 / 2}(2-2 e) d}{2} .
\end{aligned}
$$

Hence, $G$ is nonsingular if and only if $d \neq 0$ and $d \neq 2 /\left(3-2 e^{1 / 2}\right)$. In this case, problem (4.16) has a unique solution for every $\left(\begin{array}{c}\varphi \\ \psi\end{array}\right) \in \mathbb{R}^{2}$, given by (see (3.4))

$$
\begin{aligned}
x(t)= & \left(d\left(1-e^{-t}\right)-d t+1\right) v_{0}+\left(1-e^{-t}\right) v_{0}^{\prime}, \quad t \in[0,1), \\
x(t)= & \left(1-e^{1-t}\right)\left(\left(e^{-1} d-d\right) v_{0}+e^{-1} v_{0}^{\prime}\right) \\
& +\left(d\left(1-e^{1-t}\right)-d(t-1)+1\right)\left(\left(\left(1-e^{-1}\right) d-d+1\right) v_{0}+\left(1-e^{-1}\right) v_{0}^{\prime}\right) \\
= & -e^{-t-1}\left(\left(\left(\left(e d-d^{2}\right) t+2 d^{2}-e d-e\right) e^{t}-e d^{2}+e d\right) v_{0}\right. \\
& \left.+\left(((e-1) d t+(2-2 e) d-e) e^{t}+\left(e^{2}-e\right) d+e\right) v_{0}^{\prime}\right), \quad t \in\left[1, \frac{3}{2}\right],
\end{aligned}
$$

where

$$
\begin{aligned}
\left(\begin{array}{l}
v_{0} \\
v_{0}^{\prime}
\end{array}\right) & =G^{-1}\left(\begin{array}{l}
\varphi \\
\psi
\end{array}\right) \\
& =\left(\begin{array}{cc}
-\frac{2(\sqrt{e}-1) d+2}{(2 \sqrt{e}-3) d^{2}+2 d} & -\frac{(\sqrt{e}-2) d+2}{(2 \sqrt{e}-3) d^{2}+2 d} \\
-\frac{2(\sqrt{e}-1) d-2 e+2}{(\sqrt{e}(2 e-2)+(3-3 e)) d+2 e-2} & -\frac{(\sqrt{e}-2) d+2 \sqrt{e} e-2 e+2}{(\sqrt{e}(2 e-2)+(3-3 e)) d+2 e-2}
\end{array}\right)\left(\begin{array}{l}
\varphi \\
\psi
\end{array}\right),
\end{aligned}
$$

that is,

$$
\begin{aligned}
& v_{0}=\frac{-(2(\sqrt{e}-1) d+2) \varphi-((\sqrt{e}-2) d+2) \psi}{(2 \sqrt{e}-3) d^{2}+2 d} \\
& v_{0}^{\prime}=\frac{-2((\sqrt{e}-1) d-2 e+2) \varphi-((\sqrt{e}-2) d+2 \sqrt{e} e-2 e+2)}{(\sqrt{e}(2 e-2)+(3-3 e)) d+2 e-2} .
\end{aligned}
$$


For the particular case where $d=2, \varphi=1$, and $\psi=-1$, then

$$
\begin{aligned}
\left(\begin{array}{c}
v_{0} \\
v_{0}^{\prime}
\end{array}\right) & =G^{-1}\left(\begin{array}{c}
1 \\
-1
\end{array}\right) \\
& =\left(\begin{array}{cc}
-\frac{2 \sqrt{e}-1}{4 \sqrt{e}-4} & -\frac{1}{4} \\
-\frac{-e+2 \sqrt{e}-1}{\sqrt{e}(2 e-2)-2 e+2} & -\frac{e+1}{2 e-2}
\end{array}\right)\left(\begin{array}{c}
1 \\
-1
\end{array}\right)=\left(\begin{array}{c}
-\frac{1}{4} \frac{\sqrt{e}}{\sqrt{e}-1} \\
1 \frac{\sqrt{e}}{2} \frac{\sqrt{e}-1}{\sqrt{e}-1}
\end{array}\right),
\end{aligned}
$$

and hence the unique solution to

$$
\begin{aligned}
x^{\prime \prime}(t)+x^{\prime}(t)+2 x([t])=0, \quad t \in J=\left[0, \frac{3}{2}\right], \\
x\left(\frac{3}{2}\right)=x\left(\frac{1}{2}\right)+1 \\
x^{\prime}\left(\frac{3}{2}\right)=x^{\prime}\left(\frac{1}{2}\right)-1,
\end{aligned}
$$

is given by

$$
\begin{aligned}
x(t)= & \frac{\sqrt{e}(2 t-1)-2 e t+e}{-4 e+8 \sqrt{e}-4}, \quad t \in[0,1) \\
x(t)= & -\frac{\sqrt{e}\left(\left(\left(2 e^{2}+6 e\right) t-7 e^{2}-21 e\right) e^{t}+4 e^{3}+12 e^{2}\right)}{\left(4 e^{2}+24 e+4\right) e^{t+1 / 2}+\left(-16 e^{2}-16 e\right) e^{t}} \\
& -\frac{\left(\left(-6 e^{2}-2 e\right) t+21 e^{2}+7 e\right) e^{t}-12 e^{3}-4 e^{2}}{\left(4 e^{2}+24 e+4\right) e^{t+1 / 2}+\left(-16 e^{2}-16 e\right) e^{t}}, \quad t \in\left[1, \frac{3}{2}\right] .
\end{aligned}
$$

See Figure 1.

If $d=0$, then $H(s)=\left(\begin{array}{cc}1 & 1-e^{-s} \\ 0 & e^{-s}\end{array}\right)$ and the matrix

$$
G=\left[H(T-[T]) C^{[T]-[\mu]}-H(\mu-[\mu])\right] C^{[\mu]}=H\left(\frac{1}{2}\right)[C-I]
$$

is reduced to $G=\left(\begin{array}{cc}0 & e^{-3 / 2}(e-1) \\ 0 & -e^{-3 / 2}(e-1)\end{array}\right)$, which has rank one. Since $G$ is singular, there exists an infinite number of solutions to problem $(4.16)$ for every $\left(\begin{array}{c}\varphi \\ \psi\end{array}\right)$ in the image of the mapping given by the matrix $G$, that is, for every $\left(\begin{array}{c}\varphi \\ -\varphi\end{array}\right)$, where $\varphi \in \mathbb{R}$. On each of these cases, the solutions 


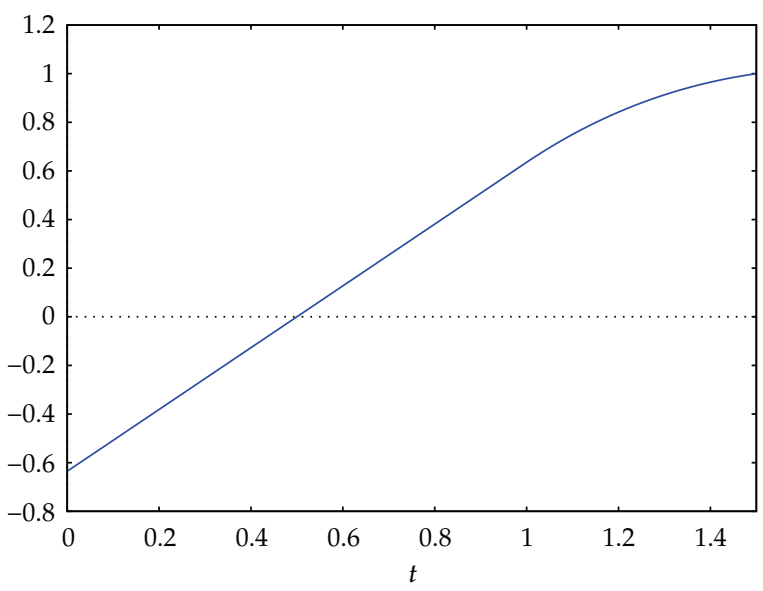

Figure 1: Solution to problem (4.23).

are given by (3.4) taking as initial position and initial slope any preimage of $\left(\begin{array}{c}\varphi \\ -\varphi\end{array}\right)$, that is, the initial slope can be taken as

$$
v_{0}^{\prime}=\frac{e^{3 / 2} \varphi}{e-1}
$$

and the initial position can be chosen as any real number $v_{0}$.

Hence, the solutions to the problem

$$
\begin{aligned}
x^{\prime \prime}(t)+x^{\prime}(t) & =0, \quad t \in J=\left[0, \frac{3}{2}\right], \\
x\left(\frac{3}{2}\right) & =x\left(\frac{1}{2}\right)+\varphi \\
x^{\prime}\left(\frac{3}{2}\right) & =x^{\prime}\left(\frac{1}{2}\right)-\varphi
\end{aligned}
$$

where $\varphi \in \mathbb{R}$, are given by

$$
x(t)=v_{0}+\left(1-e^{-t}\right) v_{0}^{\prime}, \quad t \in[0,1),
$$

and the same expression

$$
\begin{aligned}
x(t) & =\left(\begin{array}{ll}
1 & \left(1-e^{-(t-1)}\right)
\end{array}\right)\left(\begin{array}{cc}
1 & 1-e^{-1} \\
0 & e^{-1}
\end{array}\right)\left(\begin{array}{l}
v_{0} \\
v_{0}^{\prime}
\end{array}\right) \\
& =v_{0}+\left(1-e^{-t}\right) v_{0}^{\prime}, \quad t \in\left[1, \frac{3}{2}\right],
\end{aligned}
$$




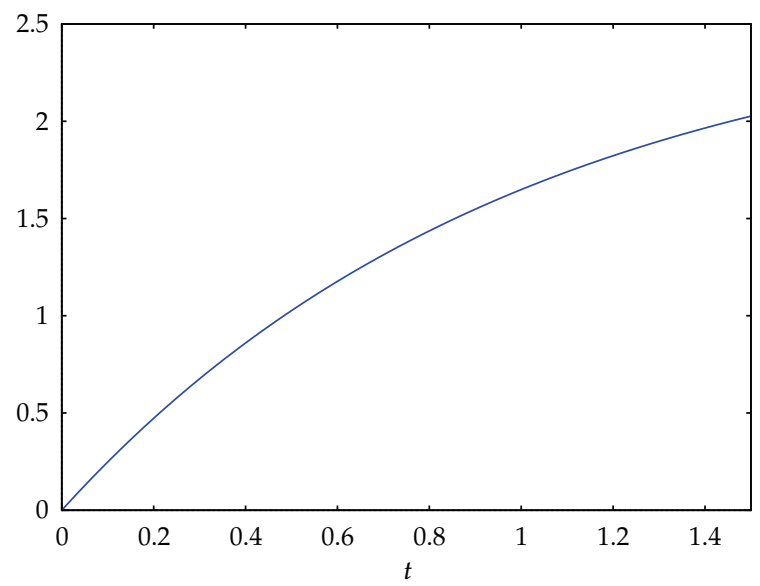

Figure 2: Solution which generates by vertical shifts the remaining solutions to problem (4.27) with $\varphi=1$.

where $v_{0} \in \mathbb{R}$ and $v_{0}^{\prime}=e^{3 / 2} \varphi /(e-1)$. Hence, for each $\varphi$ fixed, all the vertical shifts of the function $x(t)=\left(1-e^{-t}\right)\left(e^{3 / 2} \varphi /(e-1)\right), t \in[0,3 / 2]$ are solutions to (4.27). In Figure 2, we show the graph of a solution which generates, by vertical shifting, all the solutions to problem (4.27) for $\varphi=1$.

If $\psi \neq-\varphi$, there is no solution for problem (4.16) with $d=0$.

Finally, if $d=2 /\left(3-2 e^{1 / 2}\right)$, then

$$
\begin{aligned}
H(s) & =\left(\begin{array}{cc}
\frac{5-2 \sqrt{e}-2 s-2 e^{-s}}{3-2 \sqrt{e}} & 1-e^{-s} \\
\frac{2\left(e^{-s}-1\right)}{3-2 \sqrt{e}} & e^{-s}
\end{array}\right), \\
G & =H\left(\frac{1}{2}\right)[C-I] \\
& =\left(\begin{array}{cc}
\frac{4-2 \sqrt{e}-2 e^{-1 / 2}}{3-2 \sqrt{e}} & 1-e^{-1 / 2} \\
\frac{2\left(e^{-1 / 2}-1\right)}{3-2 \sqrt{e}} & e^{-1 / 2}
\end{array}\right)\left(\begin{array}{cc}
\frac{-2 e^{-1}}{3-2 \sqrt{e}} & 1-e^{-1} \\
\frac{2\left(e^{-1}-1\right)}{3-2 \sqrt{e}} & e^{-1}-1
\end{array}\right) \\
& =\left(\begin{array}{c}
\frac{-2 e^{-3 / 2}+2 e^{-1}+6 e^{-1 / 2}-10+4 \sqrt{e}}{(3-2 \sqrt{e})^{2}} \frac{-e^{-3 / 2}+e^{-1}+e^{-1 / 2}-1}{3-2 \sqrt{e}} \\
\frac{2 e^{-3 / 2}-6 e^{-1 / 2}+4}{(3-2 \sqrt{e})^{2}}
\end{array}\right)
\end{aligned}
$$


has rank one. Hence problem (4.16), for $d=2 /\left(3-2 e^{1 / 2}\right)$, has an infinite number of solutions for $\left(\begin{array}{c}\varphi \\ \psi\end{array}\right)$ in the image of the mapping given by the matrix $G$, that is, for the values of $\varphi$, and $\psi$ satisfying the system

$$
\begin{gathered}
\left(-2 e^{-3 / 2}+2 e^{-1}+6 e^{-1 / 2}-10+4 \sqrt{e}\right) v_{0}+\left(-3 e^{-3 / 2}+5 e^{-1}+e^{-1 / 2}-5+2 e^{1 / 2}\right) v_{0}^{\prime}=\varphi(3-2 \sqrt{e})^{2} \\
\left(2 e^{-3 / 2}-6 e^{-1 / 2}+4\right) v_{0}+\left(3 e^{-3 / 2}-3 e^{-1 / 2}-2 e^{-1}+2\right) v_{0}^{\prime}=\psi(3-2 \sqrt{e})^{2}
\end{gathered}
$$

for some values of $v_{0}, v_{0}^{\prime} \in \mathbb{R}$. For the existence of these values of $v_{0}, v_{0}^{\prime}$, it is necessary and sufficient that the rank of the matrix of the system (equal to one) coincides with the rank of the matrix

$$
\left(\begin{array}{ccc}
-2 e^{-3 / 2}+2 e^{-1}+6 e^{-1 / 2}-10+4 \sqrt{e} & -3 e^{-3 / 2}+5 e^{-1}+e^{-1 / 2}-5+2 e^{1 / 2} & \varphi(3-2 \sqrt{e})^{2} \\
2 e^{-3 / 2}-6 e^{-1 / 2}+4 & 3 e^{-3 / 2}-3 e^{-1 / 2}-2 e^{-1}+2 & \psi(3-2 \sqrt{e})^{2}
\end{array}\right)
$$

that is, for instance,

$$
\operatorname{det}\left(\begin{array}{cc}
-3 e^{-3 / 2}+5 e^{-1}+e^{-1 / 2}-5+2 e^{1 / 2} & \varphi \\
3 e^{-3 / 2}-3 e^{-1 / 2}-2 e^{-1}+2 & \psi
\end{array}\right)=0
$$

equivalent to

$$
\psi=\frac{3 e^{-3 / 2}-3 e^{-1 / 2}-2 e^{-1}+2}{-3 e^{-3 / 2}+5 e^{-1}+e^{-1 / 2}-5+2 e^{1 / 2}} \varphi
$$

Under this assumption, there exists an infinite number of solutions to problem

$$
\begin{aligned}
x^{\prime \prime}(t)+x^{\prime}(t)+\frac{2}{3-2 e^{1 / 2}} x([t])=0, \quad t \in J=\left[0, \frac{3}{2}\right], \\
x\left(\frac{3}{2}\right)=x\left(\frac{1}{2}\right)+\varphi, \\
x^{\prime}\left(\frac{3}{2}\right)=x^{\prime}\left(\frac{1}{2}\right)+\psi,
\end{aligned}
$$


which are given by

$$
\begin{aligned}
x(t)= & \frac{5-2 \sqrt{e}-2 t-2 e^{-t}}{3-2 \sqrt{e}} v_{0}+\left(1-e^{-t}\right) v_{0}^{\prime}, \quad t \in[0,1) \\
x(t)= & \left(\frac{5-2 \sqrt{e}-2(t-1)-2 e^{-(t-1)}}{3-2 \sqrt{e}} 1-e^{-(t-1)}\right)\left(\begin{array}{cc}
\frac{3-2 \sqrt{e}-2 e^{-1}}{3-2 \sqrt{e}} & 1-e^{-1} \\
\frac{2\left(e^{-1}-1\right)}{3-2 \sqrt{e}} & e^{-1}
\end{array}\right)\left(\begin{array}{c}
v_{0} \\
v_{0}^{\prime}
\end{array}\right) \\
= & \frac{-2 e^{-t}+4 e^{1 / 2-t}+4 \sqrt{e} t+4 e^{-1} t-6 t+4 e-16 \sqrt{e}-8 e^{-1}+15}{(3-2 \sqrt{e})^{2}} v_{0} \\
& +\frac{-e^{-t}-2 e^{1-t}+2 e^{1 / 2-t}+2 e^{-1} t-2 t-2 \sqrt{e}-4 e^{-1}+7}{3-2 \sqrt{e}} v_{0}^{\prime}, \quad t \in\left[1, \frac{3}{2}\right]
\end{aligned}
$$

where $v_{0}$ and $v_{0}^{\prime}$ satisfy (one of) the equations in (4.31).

On the other hand, if (4.34) fails, there is no solution to (4.35).

Example 4.2. Next, consider the problem

$$
\begin{aligned}
x^{\prime \prime}(t)+c x^{\prime}([t])+d x([t])=0, \quad t \in J=\left[0, \frac{3}{2}\right], \\
x\left(\frac{3}{2}\right)=x\left(\frac{1}{2}\right)+\varphi, \\
x^{\prime}\left(\frac{3}{2}\right)=x^{\prime}\left(\frac{1}{2}\right)+\psi,
\end{aligned}
$$

where $c, d, \varphi, \psi \in \mathbb{R}$. In this case, $a=b=0, T=3 / 2$, and $\mu=1 / 2$. Hence, the matrix

$$
G=\left[H(T-[T]) C^{[T]-[\mu]}-H(\mu-[\mu])\right] C^{[\mu]}=H\left(\frac{1}{2}\right)[C-I]
$$

is reduced to

$$
G=\left(\begin{array}{cc}
\frac{d^{2}+(2 c-16) d}{16} & \frac{(c-2) d+2 c^{2}-16 c+16}{16} \\
\frac{d^{2}+(2 c-4) d}{4} & \frac{(c-2) d+2 c^{2}-4 c}{4}
\end{array}\right)
$$


whose determinant is $\operatorname{det}(G)=d((d+8-4 c) / 8)$. If $d \neq 0$ and $d \neq 4 c-8$, then $G$ is invertible and problem (4.37) has a unique solution for every $\left(\begin{array}{c}\varphi \\ \psi\end{array}\right) \in \mathbb{R}^{2}$. Since

$$
G^{-1}=\frac{1}{d-4 c+8}\left(\begin{array}{cc}
\frac{(2 c-4) d+4 c^{2}-8 c}{d} & -\frac{(c-2) d+2 c^{2}-16 c+16}{2 d} \\
-(2 d+4 c-8) & \frac{d+2 c-16}{2}
\end{array}\right),
$$

the unique solution to (4.37), for $\left(\begin{array}{c}\varphi \\ \psi\end{array}\right)$ fixed, is given by

$$
\begin{aligned}
v(t)= & \left(1-\frac{d}{2} t^{2}\right) v_{0}+\left(t-\frac{c}{2} t^{2}\right) v_{0}^{\prime}, \quad t \in[0,1) \\
v(t)= & \left(1-\frac{d}{2}(t-1)^{2}(t-1)-\frac{c}{2}(t-1)^{2}\right)\left(\begin{array}{cc}
1-\frac{d}{2} & 1-\frac{c}{2} \\
-d & 1-c
\end{array}\right)\left(\begin{array}{c}
v_{0} \\
v_{0}^{\prime}
\end{array}\right) \\
= & \left(\left(1-\frac{d}{2}(t-1)^{2}\right)\left(1-\frac{d}{2}\right)+\left((t-1)-\frac{c}{2}(t-1)^{2}\right)(-d)\right) v_{0} \\
& +\left(\left(1-\frac{d}{2}(t-1)^{2}\right)\left(1-\frac{c}{2}\right)+\left((t-1)-\frac{c}{2}(t-1)^{2}\right)(1-c)\right) v_{0}^{\prime}, \quad t \in\left[1, \frac{3}{2}\right],
\end{aligned}
$$

where

$$
\begin{aligned}
& v_{0}=\frac{(2 c-4) d+4 c^{2}-8 c}{d(d+8-4 c)} \varphi-\frac{(c-2) d+2 c^{2}-16 c+16}{2 d(d+8-4 c)} \psi, \\
& v_{0}^{\prime}=-\frac{2 d+4 c-8}{d-4 c+8} v_{0}+\frac{d+2 c-16}{2(d-4 c+8)} v_{0}^{\prime} .
\end{aligned}
$$

For instance, for $c=2$ and $d=1$, then $H(s)=\left(\begin{array}{cc}1-s^{2} / 2 & s-s^{2} \\ -s & 1-2 s\end{array}\right)$ and $G=\left(\begin{array}{cc}-11 / 16 & -1 / 2 \\ 1 / 4 & 0\end{array}\right)$, which is invertible with inverse $G^{-1}=\left(\begin{array}{cc}0 & 4 \\ -2 & -11 / 2\end{array}\right)$. Then the unique solution to (4.37), for $c=2$ and $d=1$ and $\left(\begin{array}{l}\varphi \\ \psi\end{array}\right)$ fixed, is given by

$$
\begin{aligned}
& v(t)=2\left(t^{2}-t\right) \varphi+\frac{1}{2}\left(7 t^{2}-11 t+8\right) \psi, \quad t \in[0,1) \\
& v(t)=-\frac{1}{2}\left(4 t^{2}-12 t+8\right) \varphi+\frac{1}{2}\left(-5 t^{2}+13 t-4\right) \psi, \quad t \in\left[1, \frac{3}{2}\right] .
\end{aligned}
$$

See Figure 3 for the solution to (4.37) with $c=2, d=1, \varphi=1$, and $\psi=-1$ :

$$
v(t)= \begin{cases}\frac{1}{2}\left(-3 t^{2}+7 t-8\right), & t \in[0,1) \\ \frac{1}{2}\left(t^{2}-t-4\right), & t \in\left[1, \frac{3}{2}\right]\end{cases}
$$




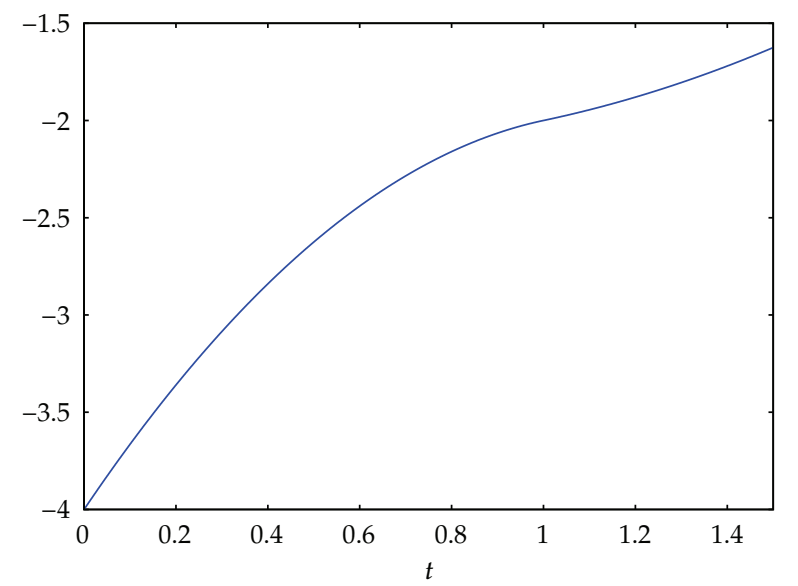

Figure 3: Solution to (4.37) for $c=2, d=1, \varphi=1$, and $\psi=-1$.

On the other hand, if $d=0$, then

$$
\begin{aligned}
H(s) & =\left(\begin{array}{cc}
1 & s-\frac{c s^{2}}{2} \\
0 & 1-c s
\end{array}\right), \\
G & =H\left(\frac{1}{2}\right)[C-I]=\left(\begin{array}{ll}
0 & \frac{c^{2}-8 c+8}{8} \\
0 & \frac{c^{2}-2 c}{2}
\end{array}\right)
\end{aligned}
$$

is singular with rank 1 , since $G_{12}=0$ if and only if $c=4 \pm 2 \sqrt{2}$ and $G_{22}=0$ if and only if $c=0$ or $c=2$.

Hence, there exists an infinite number of solutions to problem (4.37) with $d=0$, for every $\left(\begin{array}{c}\varphi \\ \psi\end{array}\right)$ in the image of the mapping given by the matrix $G$, that is, for every $\left(\begin{array}{c}\varphi \\ \psi\end{array}\right) \in \mathbb{R}^{2}$ such that $4\left(c^{2}-2 c\right) \varphi=\left(c^{2}-8 c+8\right) \psi$. Under this assumption, the solutions to (4.37) with $d=0$ are given by

$$
\begin{aligned}
v(t) & =v_{0}+\left(t-\frac{c}{2} t^{2}\right) v_{0}^{\prime}, \quad t \in[0,1) \\
v(t) & =\left(\begin{array}{ll}
1 & (t-1)-\frac{c}{2}(t-1)^{2}
\end{array}\right)\left(\begin{array}{cc}
1 & 1-\frac{c}{2} \\
0 & 1-c
\end{array}\right)\left(\begin{array}{c}
v_{0} \\
v_{0}^{\prime}
\end{array}\right) \\
& =v_{0}+\left(1-\frac{c}{2}+(1-c)\left(t-1-\frac{c}{2}(t-1)^{2}\right)\right) v_{0}^{\prime}, \quad t \in\left[1, \frac{3}{2}\right],
\end{aligned}
$$


where the initial position $v_{0}$ can be chosen as any real number and the initial slope must satisfy

$$
\begin{aligned}
& v_{0}^{\prime}=\frac{8 \varphi}{c^{2}-8 c+8}, \quad \text { if } c \neq 4 \pm 2 \sqrt{2} \\
& v_{0}^{\prime}=\frac{2 \psi}{c^{2}-2 c}, \quad \text { if } c \neq 0, c \neq 2 .
\end{aligned}
$$

For instance, if $d=0$ and $c=1$, then $H(s)=\left(\begin{array}{cc}1 & s-s^{2} / 2 \\ 0 & 1-s\end{array}\right)$, so that $G=\left(\begin{array}{cc}0 & 1 / 8 \\ 0 & -1 / 2\end{array}\right)$. Therefore, there exists an infinite number of solutions to problem (4.37) with $d=0$ and $c=1$, for every $\left(\begin{array}{l}\varphi \\ \psi\end{array}\right)$ such that $\psi=-4 \varphi$. In this case, the solutions to (4.37) with $d=0$ and $c=1$ are given by

$$
v(t)=v_{0}+ \begin{cases}8\left(t-\frac{1}{2} t^{2}\right) \varphi, & t \in[0,1) \\ 4 \varphi, & t \in\left[1, \frac{3}{2}\right]\end{cases}
$$

where $v_{0}$ is any real number. Note that $v_{0}^{\prime}=8 \varphi$. All the solutions are obtained as vertical shifts of the function

$$
f(t)= \begin{cases}8\left(t-\frac{1}{2} t^{2}\right) \varphi, & t \in[0,1) \\ 4 \varphi, & t \in\left[1, \frac{3}{2}\right] .\end{cases}
$$

See Figure 4 for the graph of function $f$ for $\varphi=1$, which generates by vertical shifting the one-dimensional space of solutions to problem (4.37) with $d=0, c=1, \varphi=1$, and $\psi=-4$.

Finally, if $d=4 c-8$, then

$$
\begin{gathered}
H(s)=\left(\begin{array}{cc}
1-(2 c-4) s^{2} & s-\frac{c}{2} s^{2} \\
(-4 c+8) s & 1-c s
\end{array}\right), \\
G=\left(\begin{array}{cc}
\frac{3 c^{2}-18 c+24}{2} & \frac{3 c^{2}-16 c+16}{8} \\
6 c^{2}-24 c+24 & \frac{3 c^{2}-10 c+8}{2}
\end{array}\right),
\end{gathered}
$$

which has rank 1 . Note that $G_{11} \neq 0$ for $c \neq 2$ and $c \neq 4 ; G_{12} \neq 0$ for $c \neq 4 / 3$ and $c \neq 4 ; G_{21} \neq 0$ for $c \neq 2$; and $G_{22} \neq 0$ for $c \neq 2$ and $c \neq 4 / 3$. 


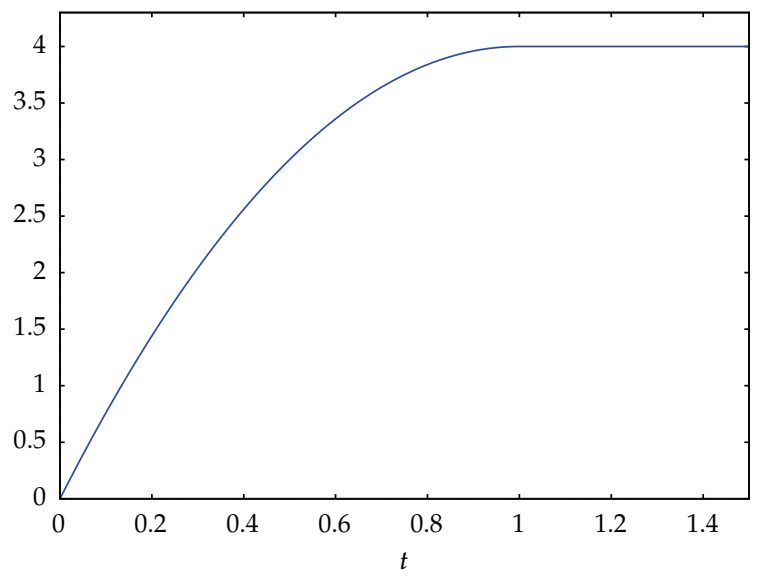

Figure 4: Solution which generates by vertical shifting the remaining solutions to problem (4.37) with $d=0, c=1, \varphi=1$, and $\psi=-4$.

Therefore, problem (4.37) for $d=4 c-8$ has solution (an infinite number of solutions) if and only if

$$
\begin{gathered}
\frac{3 c^{2}-18 c+24}{2} \psi=\left(6 c^{2}-24 c+24\right) \varphi \\
\frac{3 c^{2}-16 c+16}{8} \psi=\frac{3 c^{2}-10 c+8}{2} \varphi
\end{gathered}
$$

in which case the expressions of the solutions are given by (4.41), replacing the value of $d$, where $v_{0}$ and $v_{0}^{\prime}$ satisfy

$$
\begin{aligned}
& \frac{3 c^{2}-18 c+24}{2} v_{0}+\frac{3 c^{2}-16 c+16}{8} v_{0}^{\prime}=\varphi \\
& \left(6 c^{2}-24 c+24\right) v_{0}+\frac{3 c^{2}-10 c+8}{2} v_{0}^{\prime}=\psi
\end{aligned}
$$

Indeed,

(i) if $c=2$, then $G=\left(\begin{array}{cc}0 & -1 / 2 \\ 0 & 0\end{array}\right)$, and the problem has an infinite number of solutions for $\psi=0$ (see (4.52) since (4.51) is trivially satisfied), where $v_{0} \in \mathbb{R}$ and $v_{0}^{\prime}=-2 \varphi$ (from (4.53) since (4.54) is trivially satisfied),

(ii) if $c=4 / 3$, then $G=\left(\begin{array}{ll}8 / 3 & 0 \\ 8 / 3 & 0\end{array}\right)$, and the problem has an infinite number of solutions for $\varphi=\psi$ (see (4.51) since (4.52) is trivially satisfied), where $v_{0}=(3 / 8) \varphi=(3 / 8) \psi$ (from (4.53) or (4.54)) and $v_{0}^{\prime} \in \mathbb{R}$,

(iii) if $c \neq 2$ and $c \neq 4 / 3$, then the elements in the second row of $G$ are nonzero and, hence, the problem has an infinite number of solutions for $\varphi=\left(\left(3 c^{2}-18 c+24\right) /\left(2\left(6 c^{2}-24 c+\right.\right.\right.$ 24))) $\psi$ (from (4.51) or the same expression $\varphi=\left(\left(3 c^{2}-16 c+16\right) /\left(4\left(3 c^{2}-10 c+8\right)\right)\right) \psi$ from (4.52)). The initial position and slope are taken satisfying (4.54). 
If we take $c=4$ (see the last case distinguished), then $G=\left(\begin{array}{cc}0 & 0 \\ 24 & 16\end{array}\right)$, and conditions (4.51) and (4.52) are reduced to $\varphi=0$ and (4.54) is written as $24 v_{0}+16 v_{0}^{\prime}=\psi$.

\section{Conclusions}

In this work, we have analyzed the existence and uniqueness of solutions to the nonlocal boundary value problem for linear second-order functional differential equations with piecewise constant arguments:

$$
\begin{aligned}
x^{\prime \prime}(t)+a x^{\prime}(t)+b x(t)+c x^{\prime}([t])+d x([t])=\sigma(t), \quad t \in J=[0, T], \\
x(T)=x(\mu)+\varphi, \\
x^{\prime}(T)=x^{\prime}(\mu)+\psi,
\end{aligned}
$$

where $a, b, c, d, \varphi, \psi \in \mathbb{R}, T>0, \mu \in(0, T)$ and $\sigma \in \Lambda$, by reducing the study to the discussion of a linear system of order 2 . The nonlocal boundary conditions considered involve terms of the state function and the derivative of the state function, and the problem of interest also includes, as a particular case, periodic boundary value problems for second-order linear differential equations with functional dependence given by the greatest integer part.

Besides providing conditions on the existence and uniqueness of solutions, this approach also allows to obtain the expression of solutions explicitly, process which is illustrated with some examples.

The differences between the results in this paper and those in [19] have been included in the introduction.

\section{Acknowledgments}

The authors thank the editor and the anonymous referees for their helpful suggestions towards the improvement of the paper. This research is partially supported by Ministerio de Ciencia e Innovación and FEDER, Project MTM2010-15314.

\section{References}

[1] F. Q. Zhang, "Boundary value problems for second order differential equations with piecewise constant arguments," Annals of Differential Equations, vol. 9, no. 3, pp. 369-374, 1993.

[2] M. U. Akhmet, "On the reduction principle for differential equations with piecewise constant argument of generalized type," Journal of Mathematical Analysis and Applications, vol. 336, no. 1, pp. 646-663, 2007.

[3] M. U. Akhmet and C. Büyükadalı, "Differential equations with state-dependent piecewise constant argument," Nonlinear Analysis: Theory, Methods E Applications, vol. 72, no. 11, pp. 4200-4210, 2010.

[4] M. U. Akhmet, C. Büyükadalı, and T. Ergenç, "Periodic solutions of the hybrid system with small parameter," Nonlinear Analysis: Hybrid Systems, vol. 2, no. 2, pp. 532-543, 2008.

[5] K.-S. Chiu and M. Pinto, "Periodic solutions of differential equations with a general piecewise constant argument and applications," Electronic Journal of Qualitative Theory of Differential Equations, no. 46, pp. 1-19, 2010.

[6] M. Z. Liu, J. F. Gao, and Z. W. Yang, "Preservation of oscillations of the Runge-Kutta method for equation $x^{\prime}(t)+a x(t)+a_{1} x([t-1])=0$," Computers \& Mathematics with Applications. An International Journal, vol. 58, no. 6, pp. 1113-1125, 2009. 
[7] J. J. Nieto and R. Rodríguez-López, "Green's function for second-order periodic boundary value problems with piecewise constant arguments," Journal of Mathematical Analysis and Applications, vol. 304, no. 1, pp. 33-57, 2005.

[8] M. Pinto, "Asymptotic equivalence of nonlinear and quasi linear differential equations with piecewise constant arguments," Mathematical and Computer Modelling, vol. 49, no. 9-10, pp. 1750-1758, 2009.

[9] G. Seifert, "Second order scalar functional differential equations with piecewise constant arguments," Journal of Difference Equations and Applications, vol. 8, no. 5, pp. 427-445, 2002.

[10] G. Seifert, "Second-order neutral delay-differential equations with piecewise constant time dependence," Journal of Mathematical Analysis and Applications, vol. 281, no. 1, pp. 1-9, 2003.

[11] P. Yang, Y. Liu, and W. Ge, "Green's function for second order differential equations with piecewise constant arguments," Nonlinear Analysis: Theory, Methods \& Applications, vol. 64, no. 8, pp. 1812-1830, 2006.

[12] Z. W. Yang, M. Z. Liu, and J. J. Nieto, "Runge-Kutta methods for first-order periodic boundary value differential equations with piecewise constant arguments," Journal of Computational and Applied Mathematics, vol. 233, no. 4, pp. 990-1004, 2009.

[13] R. Yuan, "Pseudo-almost periodic solutions of second-order neutral delay differential equations with piecewise constant argument," Nonlinear Analysis: Theory, Methods E Applications, vol. 41, no. 7-8, pp. 871-890, 2000.

[14] R. Yuan, "On the second-order differential equation with piecewise constant argument and almost periodic coefficients," Nonlinear Analysis: Theory, Methods E Applications, vol. 52, no. 5, pp. 1411-1440, 2003.

[15] A. Cabada, J. B. Ferreiro, and J. J. Nieto, “Green's function and comparison principles for first order periodic differential equations with piecewise constant arguments," Journal of Mathematical Analysis and Applications, vol. 291, no. 2, pp. 690-697, 2004.

[16] J. J. Nieto, "A comparison result for a linear differential equation with piecewise constant delay," Glasnik Matematički. Serija III, vol. 39(59), no. 1, pp. 73-76, 2004.

[17] Y. Liu, P. Yang, and W. Ge, "Periodic solutions of higher-order delay differential equations," Nonlinear Analysis: Theory, Methods \& Applications, vol. 63, no. 1, pp. 136-152, 2005.

[18] L. Pan, "Periodic solutions for higher order differential equations with deviating argument," Journal of Mathematical Analysis and Applications, vol. 343, no. 2, pp. 904-918, 2008.

[19] R. Rodríguez-López, "Nonlocal boundary value problems for second-order functional differential equations," Nonlinear Analysis: Theory, Methods \& Applications, vol. 74, no. 18, pp. 7226-7239, 2011. 


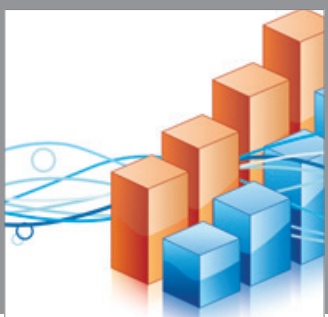

Advances in

Operations Research

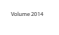

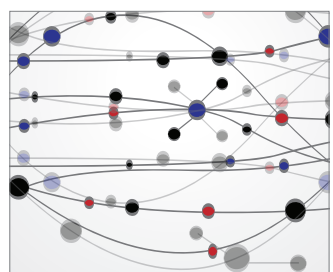

\section{The Scientific} World Journal
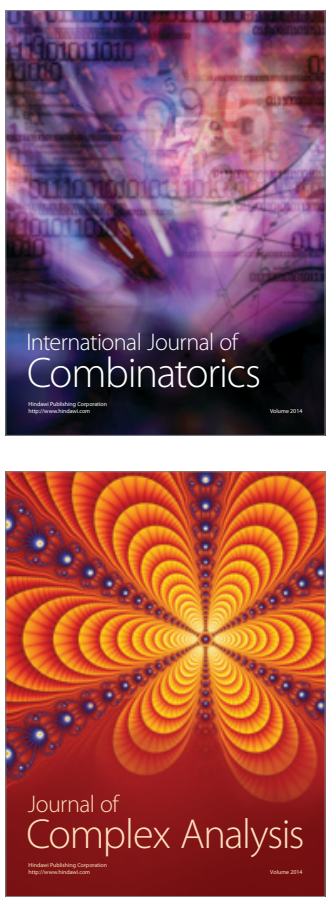

International Journal of

Mathematics and

Mathematical

Sciences
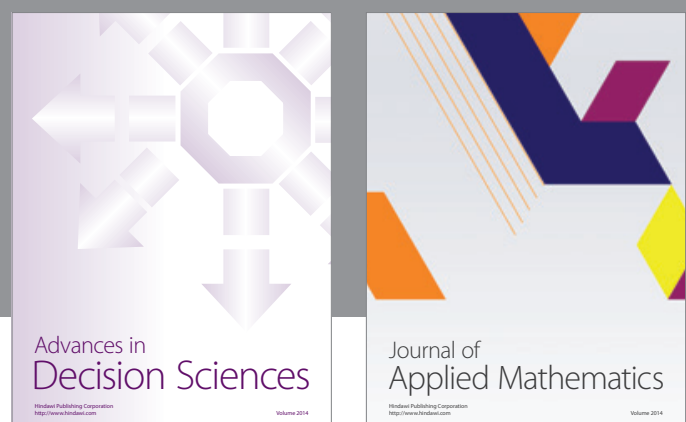

Journal of

Applied Mathematics
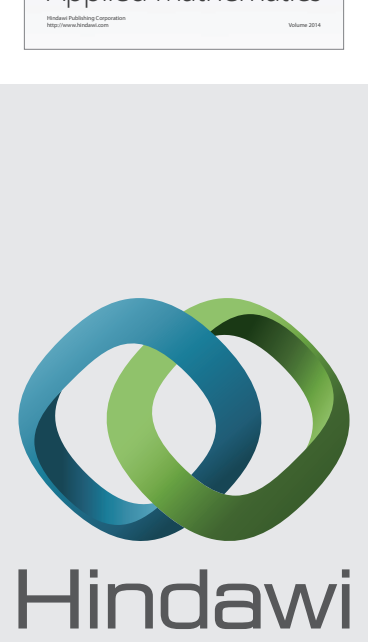

Submit your manuscripts at http://www.hindawi.com
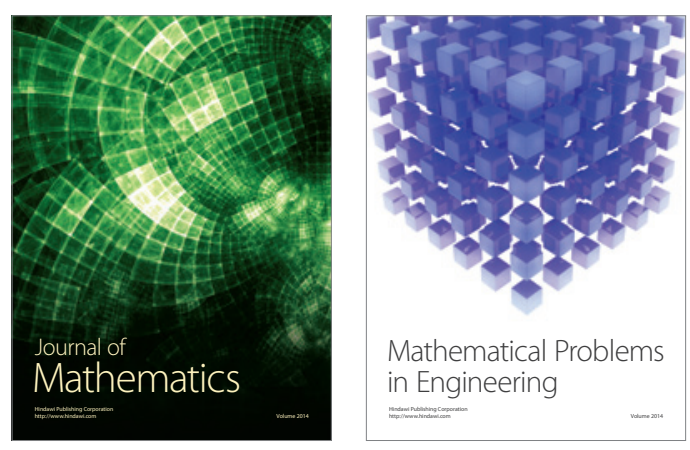

Mathematical Problems in Engineering
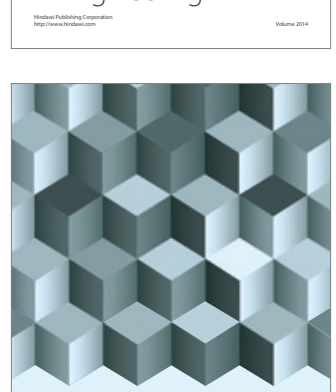

Journal of

Function Spaces
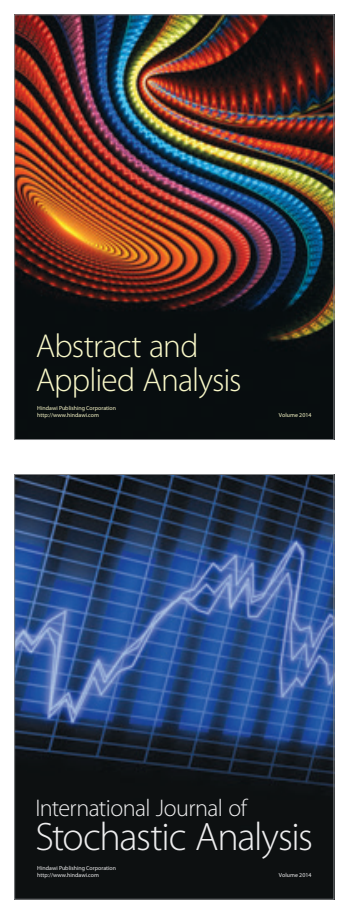

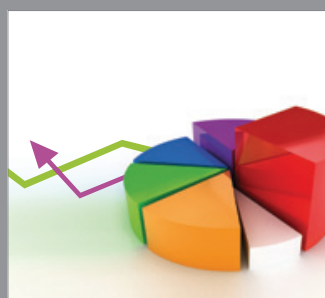

ournal of

Probability and Statistics

Promensencen
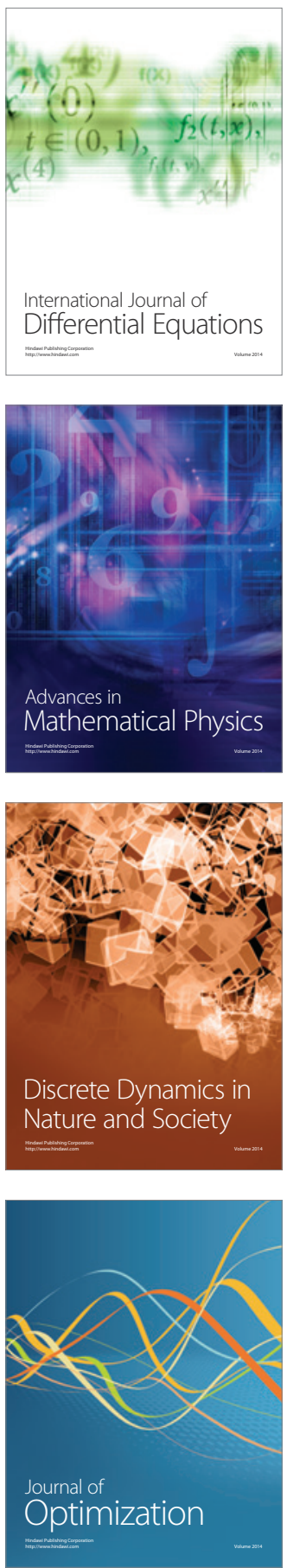